\title{
Towards Respiration Rate Monitoring Using an In-Ear Headphone Inertial Measurement Unit
}

\author{
Tobias Röddiger, Daniel Wolffram, David Laubenstein, Matthias Budde and Michael Beigl \\ \{roeddiger,wolffram,budde,beigl\}@teco.edu, ${ }^{*}$ \{david.laubenstein\}@student.kit.edu \\ Karlsruhe Institute of Technology \\ Karlsruhe, Germany
}

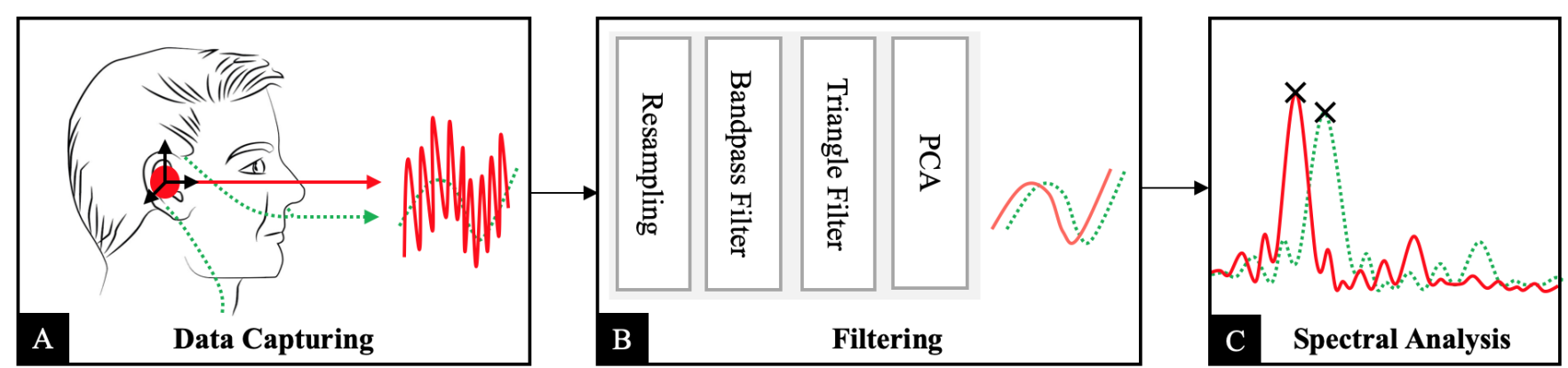

Figure 1: To measure respiration with an in-ear headphone IMU, we capture 3D acceleration and gyroscope data at $50 \mathrm{~Hz}(\mathrm{~A})$. We record the ground truth with a pressure transducer. Data is processed as illustrated in (B): we split the data into 20-second windows and interpolate using cubic splines, resampling at $256 \mathrm{~Hz}$. Our pipeline discards windows with too much movement. We apply a Butterworth bandpass filter to remove noise and a triangle filter for further smoothening without loss of timing information. Finally, we use FFT with zero padding in $(\mathrm{C})$ and compute the maximum to calculate the respiratory rate.

\begin{abstract}
State-of-the-art respiration tracking devices require specialized equipment, making them impractical for every day at-home respiration sensing. In this paper, we present the first system for sensing respiratory rates using in-ear headphone inertial measurement units (IMU). The approach is based on technology already available in commodity devices: the eSense headphones. Our processing pipeline combines several existing approaches to clean noisy data and calculate respiratory rates on 20 -second windows. In a study with twelve participants, we compare accelerometer and gyroscope based sensing and employ pressure-based measurement with nasal cannulas as ground truth. Our results indicate a mean absolute error of 2.62 CPM (acc) and 2.55 CPM (gyro). This overall accuracy is comparable to previous approaches using accelerometer-based sensing, but we observe a higher relative error for the gyroscope. In contrast to related work using other sensor positions, we can not report significant differences between the two modalities or the three postures standing, sitting, and lying on the back (supine). However, in general, performance varies drastically between participants.
\end{abstract}

\section{CCS CONCEPTS}

-Human-centered computing $\rightarrow$ Ubiquitous and mobile computing; $・$ Applied computing $\rightarrow$ Consumer health.

\section{KEYWORDS}

In-Ear Headphones, Respiration, Monitoring, IMU, Breathing

\section{ACM Reference Format:}

Tobias Röddiger, Daniel Wolffram, David Laubenstein, Matthias Budde and Michael Beigl. 2019. Towards Respiration Rate Monitoring Using an InEar Headphone Inertial Measurement Unit. In 1st International Workshop on Earable Computing (EarComp'19). ACM, New York, NY, USA, 6 pages. https://doi.org/10.1145/3345615.3361130

\section{INTRODUCTION}

When tracking respiration rates in day-to-day scenarios, respiratory inductance plethysmography is the current state-of-the-art. A belt straps around the user's chest and abdominal wall to measure the expansion while breathing in and out [21]. Such devices are specialized and expensive equipment and not suitable for everyday use. A different method, applied for instance in sleep labs and under medical conditions, uses nasal cannulas made from plastic tubes which redirect the airflow to pressure transducers [15]. These tubes are uncomfortable to wear as they are placed inside the nostrils and are unhygienic when used multiple times or with different users. As an alternative, we investigate respiratory monitoring based on a 6-axis inertial measurement unit (IMU) embedded into a standard in-ear headphone form factor. This technology is potentially accessible to a broad set of users, as already today, earphones with 
integrated IMUs are commercially available (like e.g., the Apple AirPods).

Embedding respiratory sensing into headphones opens up a set of use cases where auditory feedback couples to breathing. For example, Harris et al. suggest that auditory biofeedback can enable the control of the respiration rate of users [5], which can help with stress management or support guided meditations [17]. We could also detect the interruption of breathing during sleep (apnea) and alert the user after a defined time threshold. Such scenarios naturally fit our sensor setup because the user maintains a steady position which avoids movement artifacts, and the headphones seamlessly integrate wit providing audio feedback.

In this paper, we propose the use of headphones equipped with a 6-axis IMU (accelerometer and gyroscope). We present the working principle of our system and introduce a data processing pipeline. We evaluated our system in a lab study with twelve participants and compared between standing, sitting, and lying on the back (supine) as well as accelerometer and gyroscope based tracking. Our results indicate good outcomes for a subpopulation of participants.

\section{BACKGROUND AND RELATED WORK}

Previous research proposes a broad set of alternatives to the formerly mentioned state-of-the-art for respiration rate tracking.

Systems based on UWB, WiFi, or vision $[1,12,19]$ have significant advantages because they are not attached to the user. However, they require specialized setups which can be complicated or might have problems with other people present in the room. Further techniques which do not require nasal cannulas to measure respiration from human breath include gas sensors that measure, e.g., volatile organic compounds [16] or humidity [13]. They still require to be placed in the air stream or have to be attached close to the area around mouth and nose.

Acceleration and gyroscope data have significant advantages in terms of cost and unobtrusiveness because many modern devices already come equipped with these inexpensive sensors $(<5 \$$, single quantity). The idea to use the IMU's data is not new, and others have shown that the underlying principle does work. It has been implemented using chest belts [2] or smartwatches [3, 18]. Another approach which is particularly relevant because it is head-worn uses Google Glass smart glasses [7]. However, this is impractical for scenarios where glasses can not be comfortably worn, e.g., when lying on the side. Additionally, it remained questionable to us if a sensor plugged into the ear canal is capable of achieving similar results. Nevertheless, we can use their results to add context to our system's performance and draw additional conclusions. Existing work has also explored the option to support meditation sessions with breathing monitored in real-time using a so-called breathing model derived from a smartwatch's IMU [4].

Tracking respiration rates with a headset has been explored in [14] but their method requires placing the microphone of the headset under users nose. In [11] a microphone is positioned in the ear-canal using in-ear to record and interpret breathing sounds. It was tested with 25 subjects and yielded 2.7 breathing cycles per minute (CPM) absolute mean error for quiet environments, and they report problems with increasing background noises. In [6], an accelerometer-equipped device wraps around the user's ear to measure respiration. They only evaluate the device with a single participant and do not consider gyroscope data as well as an in-ear form factor.

\section{WORKING PRINCIPLE}

\subsection{System Design}

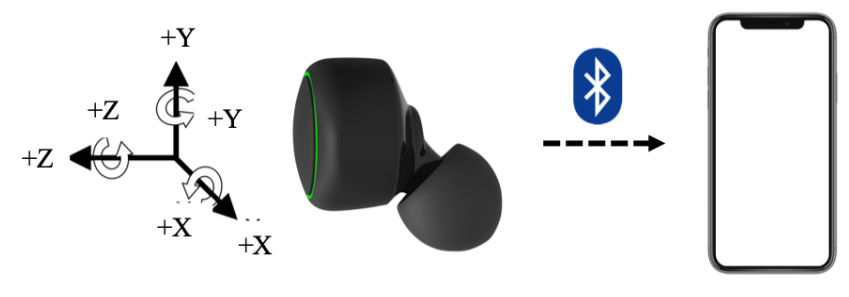

Figure 2: The Nokia Bell Labs eSense headphones [9] (left) connect via Bluetooth and transfer gyroscope and accelerometer data to the smartphone app (right).

We leverage the eSense platform [9], which has been kindly provided to us by Nokia Bell Labs. It comes equipped with a six-axis IMU in its left earbud and connects via Bluetooth Low Energy (BLE). We record the $\mathrm{x}, \mathrm{y}$ and $\mathrm{z}$ angular velocity in $\mathrm{deg} / \mathrm{s}$ using the built-in gyroscope and the $\mathrm{x}, \mathrm{y}$ and $\mathrm{z}$ acceleration in $\mathrm{m} / \mathrm{s}^{2}$ as illustrated in Figure 2. We sample at the maximum frequency of $50 \mathrm{~Hz}$ and do not use the integrated low pass filter settings. We do not record other information provided by the platform (e.g., microphone).

We have implemented a mobile application in Swift for iOS, which connects to the eSense earbuds. The data is stored locally on the phone, and timestamps are taken on a rolling basis as the Bluetooth packages arrive. To transfer the data to a computer, we export it to a CSV file and send it using any of the options provided natively by the operating systems' sharing options (in our case, AirDrop). Finally, we feed the files into our processing pipeline.

\subsection{Data Processing Pipeline}

To compute the respiration rate, we use the same steps independent from gyroscope or accelerometer. We expand upon the approach proposed in [7]. We apply steps (1), (2), (4), and (5) to each axis. Additionally, steps (3) and (5) remove motion sensitivity.

(1) To remove signal shifts and trends, a moving average window of 3 samples is subtracted from each dimension. Additionally, we apply an averaging filter with a window size of 2 seconds to each of the components, corresponding to one respiration cycle at the maximum breathing rate ( 30 breaths per minute).

(2) To inflate our data, we apply a cubic spline interpolation and resample the resulting signal at $256 \mathrm{~Hz}$. As there are small variations in timestamps due to Bluetooth latency, this also helps to create equidistant samples.

(3) Similar to [18] we discard windows if movement is too high. If $3 \%$ or more of all accelerometer data points are above our threshold of $10 \mathrm{~m} / \mathrm{s}^{2}$ the entire sequence is sorted out. Additionally, we apply hard thresholding for samples \pm 2 SD.

(4) We apply a bandpass Butterworth filter of order four and cut-off frequencies of $0.1 \mathrm{~Hz}$ and $0.5 \mathrm{~Hz}$, which removes noise and is equivalent to 6 to 30 breath cycles per minute (CPM). 

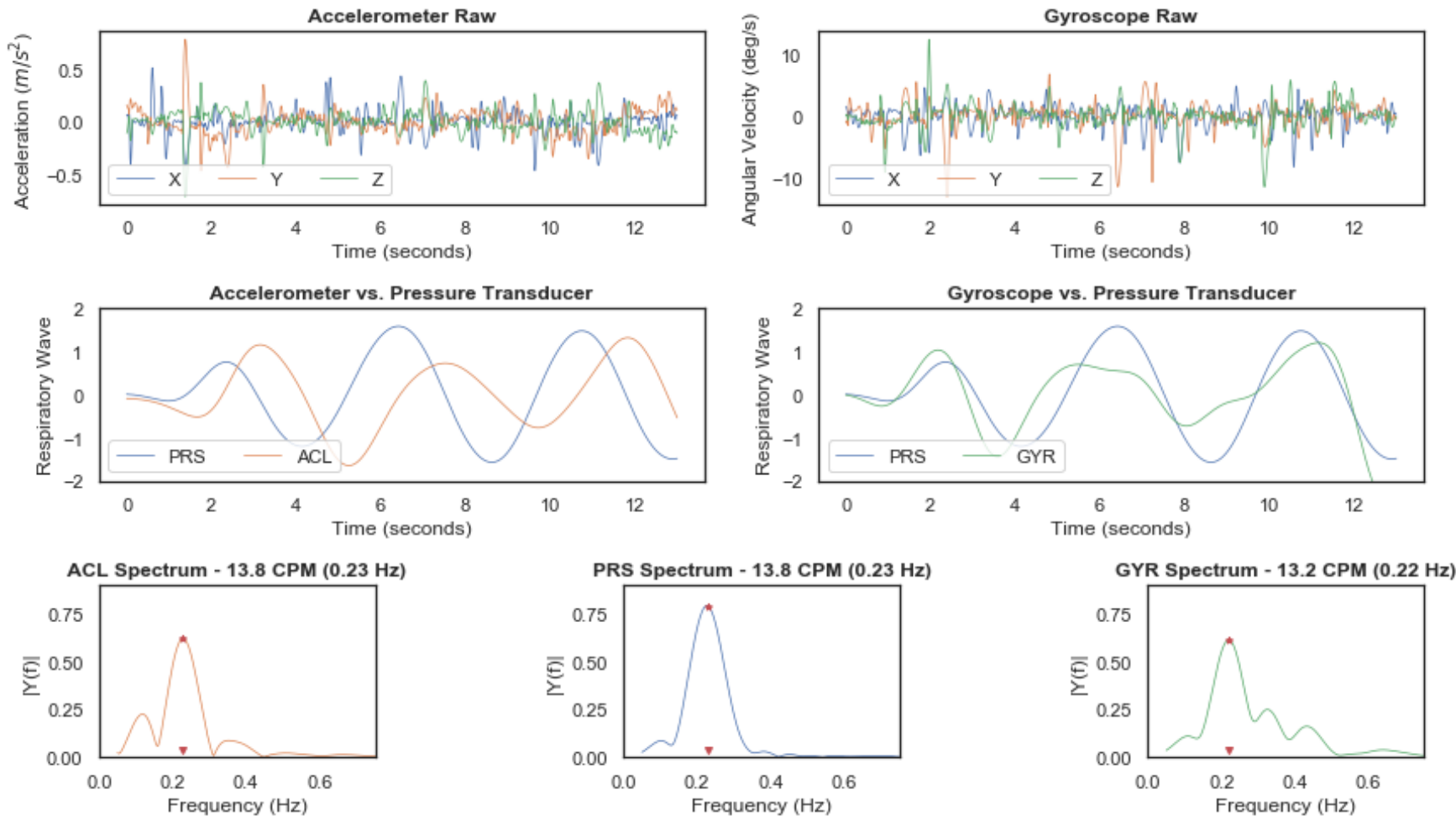

Figure 3: The graph in the top left corner shows the raw acceleration signal of the $\mathrm{X}$-, $\mathrm{Y}$ - and Z-axis, the graph in the top right the gyroscope data of the $\mathrm{X}$-, $\mathrm{Y}$ - and $\mathrm{Z}$-axis. The graph shown at the center-left displays the filtered acceleration signal compared to the ground truth and the center-right the filtered gyroscope signal compared to the ground truth. The three graphs in the bottom show the spectrum of the processed accelerometer signal (left), the ground truth pressure signal (center) and the gyroscope (right).

(5) To further smoothen the signals while retaining the peak positions, we apply a triangle filter with a width of 2 seconds as described in [4].

(6) To make the results independent from changes on different axes for different postures, we perform a principal component analysis (PCA).

(7) We perform a spectral analysis of each principal component using a Fast Fourier Transformation (FFT) with zero-padding and compute the maximum peak and its magnitude for each component. We then report the frequency corresponding to the peak with the highest magnitude as respiration frequency that we can convert to CPM.

Figure 3 displays the raw signals captured from the accelerometer (left) and gyroscope (right) of one of our study participants sitting. The two graphs indicate how noisy the initial data signals are along all axes. As the user breathes, the accelerometer signal visibly oscillates around zero on the Y-axis and the gyroscope on the Zaxis. The second row compares a normalized ground truth signal captured using nasal cannulas hooked to a pressure transducer (PRS) with our filtered signal. The bottom row displays the three different spectra computed from the respective sensor signals. A red star indicates the maximum in each spectrum, which illustrates a minimal error between the three different modalities.

\section{EVALUATION}

\subsection{Study Design}

To evaluate our system, we recruited twelve participants (two female, ten male) between the ages of 21 and 39 (mean age 26) for a lab study. The mean height was $179 \mathrm{~cm}$ and weight $81 \mathrm{~kg}$. We did not pay participants. Our experiment was conducted in a room with a couch to lie on and a stable chair with armrests for sitting down. We placed both eSense earbuds [9] into the participant's ears (left earbud equipped with the IMU) and hooked them up to nasal cannulas as ground truth. No audio was played during the study.

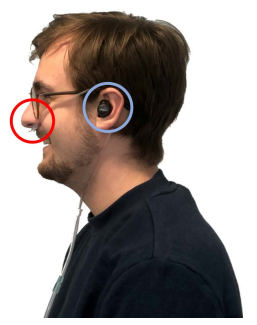

Figure 4: Participant wearing nasal cannulas (red, left circle) and the eSense headphones (blue, right circle). 

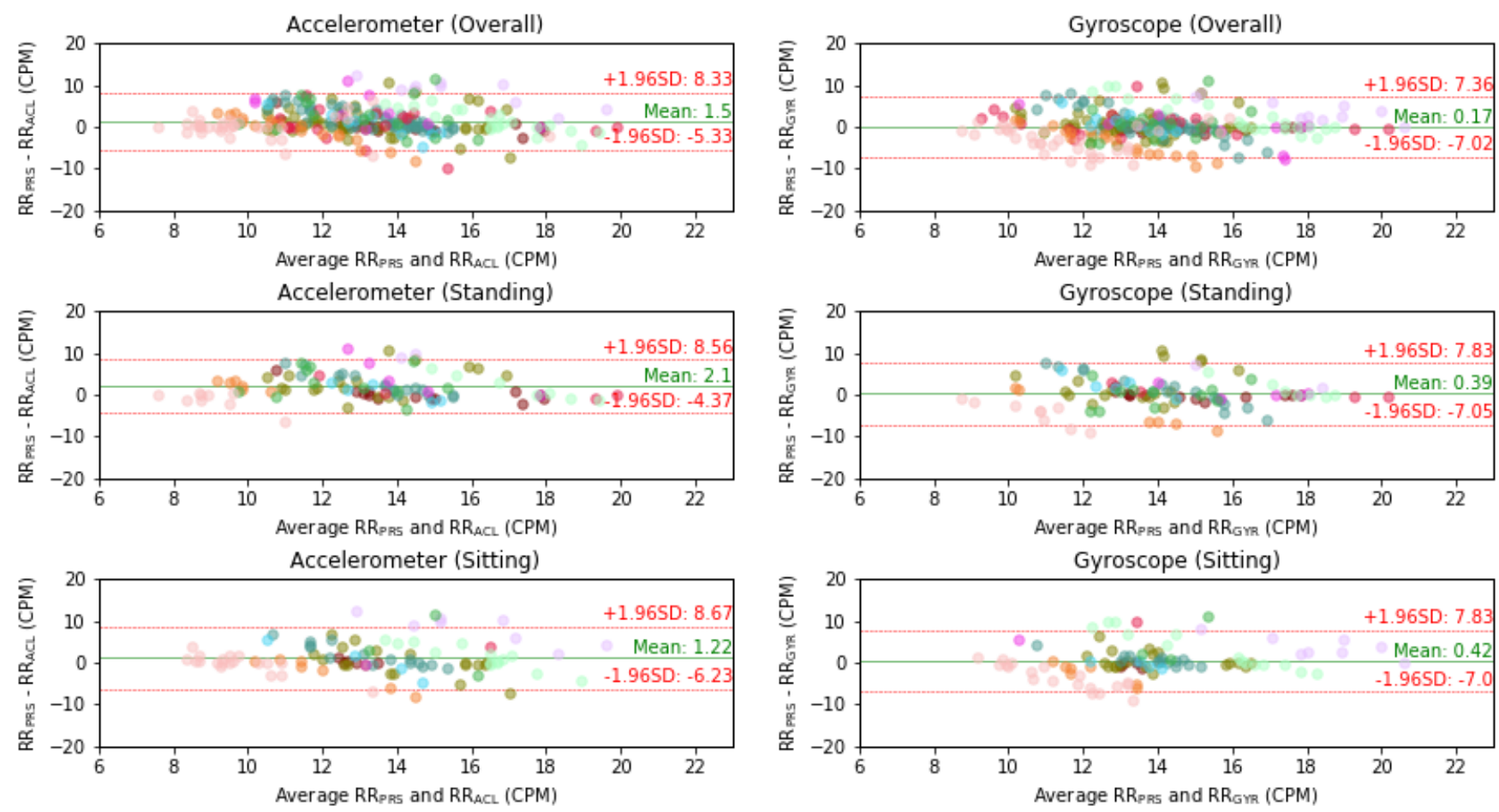

Gyroscope (Sitting)
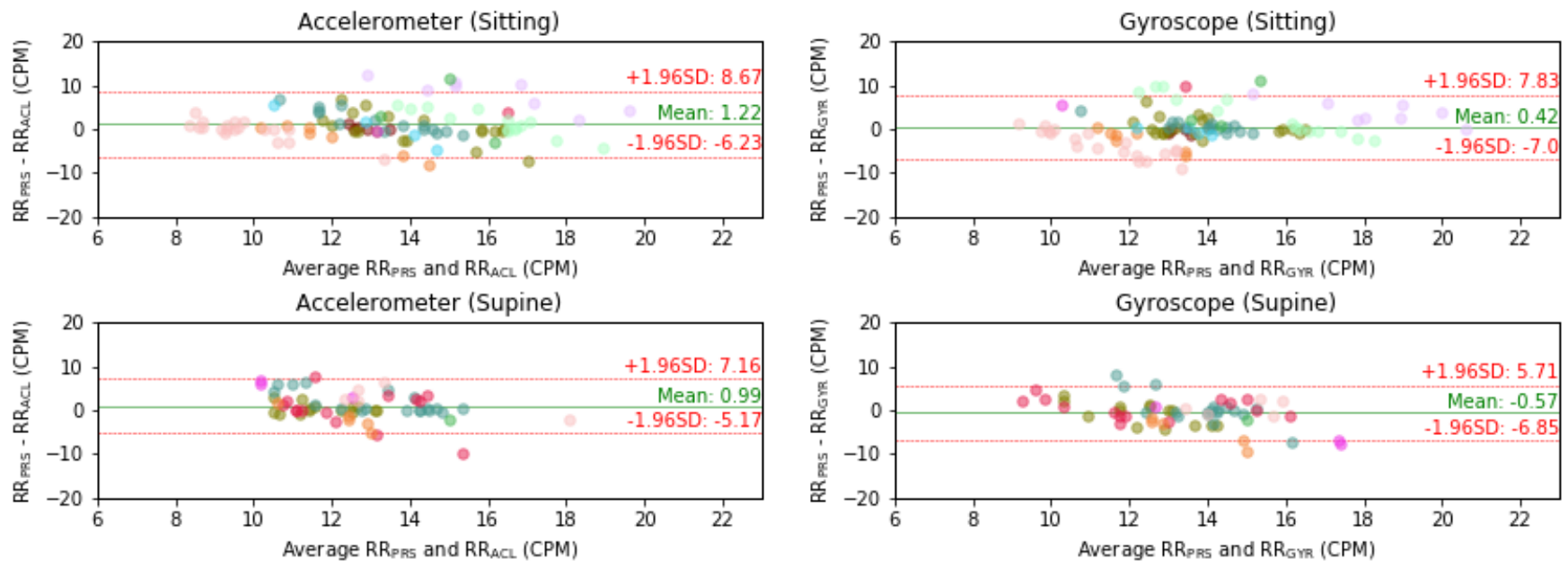

Figure 5: Shows Bland-Altman plots for the accelerometer (left) and gyroscope (right) with an aggregated graph for the overall performance and for the postures standing, sitting and lying on the back. Per-user results indicated in different colors.

Table 1: Respiration rate system performance in cycles-perminute (CPM).

\begin{tabular}{lccc}
\hline Sensor & MAE & SD & RMSE \\
\hline Accelerometer & 2.62 & 2.74 & 3.79 \\
Gyroscope & 2.55 & 2.63 & 3.67 \\
\hline
\end{tabular}

During the first phase of our evaluation, each participant was asked to breathe normally for one minute each in three different postures (standing, sitting, and lying) while otherwise keeping as still as possible. This step was followed by a second phase, in which we then asked them to perform a short 30 -second jumping jacks session before each of the three postures, which we anticipated to result in a more dynamic dataset. After performing the activity, we again recorded respiration data for one minute for every posture.

We used the Williams design generalized Latin squares [20] to balance for first-order carryover effects introduced by a potentially unnatural breathing behavior when asked to breathe on the
Table 2: Comparison between modalities and postures (MAE / SD) in cycles-per-minute (CPM).

\begin{tabular}{lccc}
\hline Sensor & Standing & Sitting & Supine \\
\hline Accelerometer & $3.15 / 2.74$ & $3.10 / 2.80$ & $2.56 / 2.19$ \\
Gyroscope & $2.45 / 2.22$ & $2.74 / 2.64$ & $2.68 / 2.00$ \\
\hline
\end{tabular}

spot. For the three different postures, this resulted in six different sequences, which we assigned to participants in a round-robin fashion according to their arrival time. The sequence for the first and second phase of the evaluation was identical within each session. After completing these tasks, participants were asked to fill in a short questionnaire, which included demographic questions (sex, age, weight, height) as well as a question inquiring whether participants felt that they had breathed naturally and space for free-text feedback.

The respiration ground truth was collected using a custom made monitoring device: We wired a RedBear BLE Nano $v 2$ to a pressure 

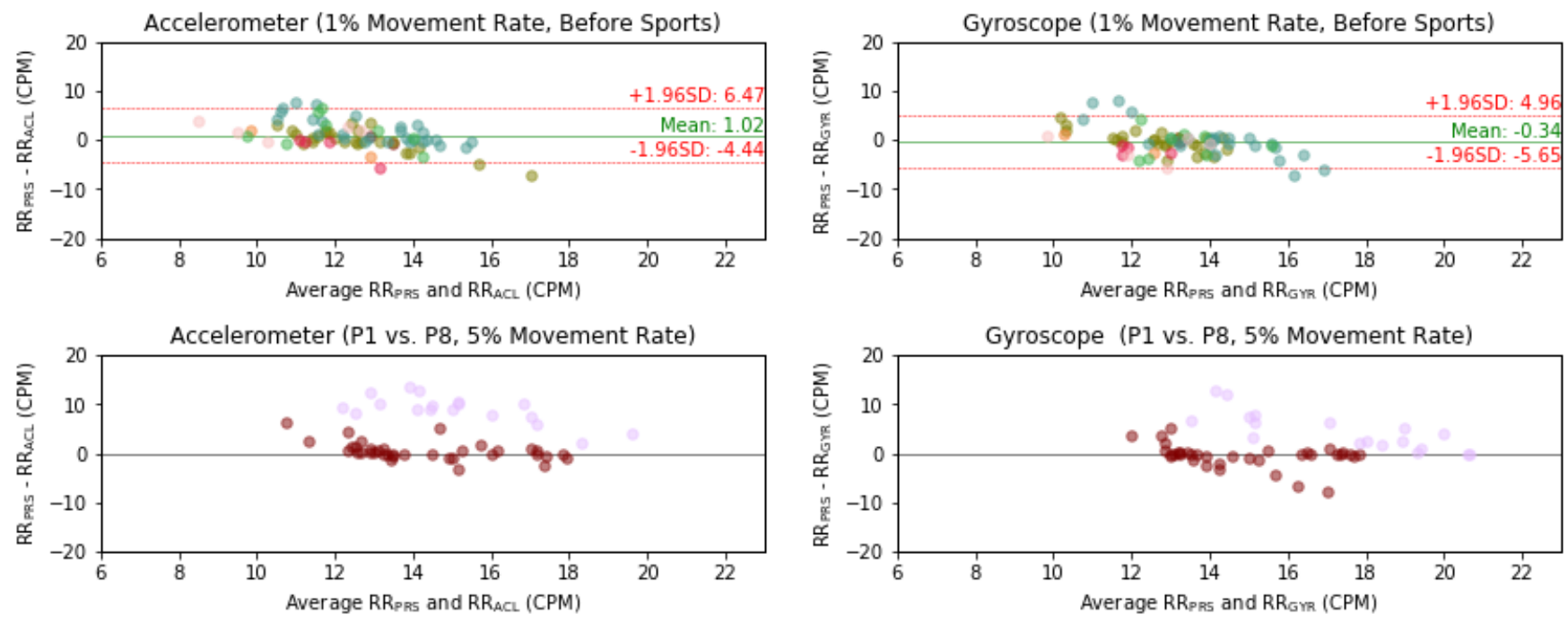

Figure 6: The two diagrams in the first row above indicate how reducing the movement threshold increases the accuracy. The diagrams in the second row compare the data of two participants, whereas P1 achieves a much lower mean error than P8 even at a higher movement threshold of $5 \%$. Per-User results indicated in different colors.

transducer that connects to nasal cannulas (see Figure 4). The device samples pressure data at a frequency of $50 \mathrm{~Hz}$. We filter the signal with the same data processing pipeline as described in 3.2, except we do not apply a PCA. It also connects to our mobile application, which we installed on an Apple iPhone $X$ for our study. Similarly to the acceleration and gyroscope data, we use the arrival time of the Bluetooth packages on the phone as timestamps, which makes it easy to synchronize the data afterward. We also attached the device on the user as displayed in Figure 4.

\subsection{Results}

In total, we collected 72 minutes of breathing data. We shift a sliding window at an interval of 5 seconds over every one-minute data frame. This process yields 669 twenty-second breathing sequences. After removing the ones with too many movement artifacts, 253 remain. Ground truth respiration rates range from 7.6 to 22 in cyclesper-minute (CPM) for those sequences. To evaluate the agreement between our approach and the ground truth measurement, we utilize Bland-Altman plots shown in Figure 5. In most settings, the observed differences are centered around zero and show no significant bias, also observable from the displayed mean error. The plots also show the limits of agreement (interval between +1.96SD and $-1.96 \mathrm{SD}$ ) that contain $95 \%$ of the measured differences. Additionally, we have computed several metrics for ease of comparison, namely the mean absolute error, its standard deviation, and the root mean squared error shown in Table 1. We further broke it down by body posture in Table 2. Overall, the performance of gyroscope is similar to the accelerometer but varies between postures. We achieve the best results for the accelerometer in the supine position, followed by similar results for sitting and standing. For gyroscope, we achieve comparable results for all three postures.

\section{DISCUSSION}

Generally, we can observe that our method is highly sensitive to motion artifacts. The first row in Figure 6 shows that we achieve better results after setting the motion threshold to $1 \%$ and limiting the dataset to non-aroused participants. Introducing this limitation reduces the MAE to 2.09 $\mathrm{CPM}$ for the accelerometer and to 1.90 $\mathrm{CPM}$ for the gyroscope. Additionally, we see significant differences between subjects. For example, the second row in Figure 6 shows that even after raising the motion threshold to $5 \%$ participant $\mathrm{P} 1$ has much better results than P8 (MAE 1.21 ACC / 1.45 GYR vs. MAE 8.97 ACC / 4.58 GYR). We do not know what causes these differences; however, bad fitting of the earplugs or differences in pose and anatomy could be a reason.

\subsection{Comparison with Related Work}

In Table 3, we compare our results to related work. Hernandez et al. [7] have evaluated smart glasses and also smartwatches [8] for the same three postures as we did. Compared to [7] we do not observe significant differences between gyroscope and accelerometer. Overall, the head seems to be a less suitable position for tracking respiration rates than e.g., the wrist. We yield higher error rates than $[7,18]$, especially for the gyroscope. The root causes for differences between the two head-worn devices are unclear.

Table 3: Performance (MAE / SD) in cycles-per-minute (CPM) of our system compared to related work.

\begin{tabular}{lccc}
\hline Sensor & In-Ear & Glasses [7] & Watch [8] \\
\hline Accelerometer & $2.62 / 2.74$ & $2.29 / 3.43$ & $0.92 / 2.20$ \\
Gyroscope & $2.55 / 2.63$ & $1.39 / 2.27$ & $0.38 / 1.19$ \\
\hline
\end{tabular}


Comparing the different poses, we have similar results to [7] for the accelerometer in the standing posture; however, perform worse for sitting and lying down. Additionally, the gyroscope's performance for standing is comparable, but we report higher errors for the sitting and supine position.

According to [10], the thoracic spine moves back and forth, whereas the spine moves up and down while breathing. The resulting motion of the head could be different when measuring above the eye compared to the ear in different poses and on varying head positions. We further theorize that the differences could be caused by a dampening effect of the ear plugs' flexible caps which might absorb motion.

\subsection{Limitations}

For our evaluation, we measured nasal respiration using a pressure transducer. After performing the physiologically straining task of jumping jacks, several participants reported the urge to breathe through the mouth afterward. We did not limit them to nose breathing before the study, but several participants reported that they "felt forced to not breathe through the mouth" (P2). An FDA-cleared chest belt based on respiratory inductance plethysmography is a more suitable methodology, which could support a more natural breathing behavior and therefore positively affect results. To identify relationships between the fit of the earplugs and respiration rate estimation accuracy, we suggest measuring ear sizes and film participants in future studies. Additionally, our experimental environment left things to explore visually (e.g., posters), which could result in additional motion artifacts.

\section{CONCLUSION AND FUTURE WORK}

In this paper, we explored the feasibility to use in-ear headphones for tracking respiratory rates and compared our results to related work. We have evaluated a data processing pipeline which combines multiple factors from previous work to fit an in-ear headphone use case. We compared accelerometer and gyroscope data and present results for the three different postures standing, sitting and lying on the back (supine). Our approach was validated by comparing measurements with ground truth data from nasal cannulas connected to a pressure transducer. In general, our results suggest that the ear is a less suitable position for measuring respiratory rates than, e.g., on the wrist. Overall, our solution has a high sensitivity to small motion artifacts. Nevertheless, we achieve stable performances for a subpopulation of participants. The underlying root causes for those differences remain unclear at this point and will be investigated in future research.

To explore the inaccuracies between subjects, we suggest to investigate the potentially loose attachment of in-ear headphones further and examine possible dampening effects created by the headphone's cushion. We suggest using different sizes of ear canal caps depending on the user's ear size. We also propose a comparison study between the ear and above eye positions for respiratory rate tracking, which could reveal further insights that cause the performance differences discovered in this paper. Finally, a more advanced approach fusing accelerometer and gyroscope data and even microphone signals are likely to yield better results.

\section{REFERENCES}

[1] Heba Abdelnasser, Khaled A Harras, and Moustafa Youssef. 2015. UbiBreathe: A ubiquitous non-invasive WiFi-based breathing estimator. In Proceedings of the 16th ACM International Symposium on Mobile Ad Hoc Networking and Computing. ACM, 277-286.

[2] Andrew Bates, Martin J Ling, Janek Mann, and DK Arvind. 2010. Respiratory rate and flow waveform estimation from tri-axial accelerometer data. In 2010 International Conference on Body Sensor Networks. IEEE, 144-150.

[3] Marian Haescher, Denys JC Matthies, John Trimpop, and Bodo Urban. 2016. SeismoTracker: upgrade any smart wearable to enable a sensing of heart rate, respiration rate, and microvibrations. In Proceedings of the 2016 CHI Conference Extended Abstracts on Human Factors in Computing Systems. ACM, 2209-2216.

[4] Tian Hao, Chongguang Bi, Guoliang Xing, Roxane Chan, and Linlin Tu. 2017. Mindfulwatch: A smartwatch-based system for real-time respiration monitoring during meditation. Proceedings of the ACM on Interactive, Mobile, Wearable and Ubiquitous Technologies 1, 3 (2017), 57.

[5] Jason Harris, Sarah Vance, Odair Fernandes, Avinash Parnandi, and Ricardo Gutierrez-Osuna. 2014. Sonic respiration: controlling respiration rate through auditory biofeedback. In CHI'14 Extended Abstracts on Human Factors in Computing Systems. ACM, 2383-2388.

[6] David Da He. 2013. A wearable heart monitor at the ear using ballistocardiogram $(B C G)$ and electrocardiogram (ECG) with a nanowatt ECG heartbeat detection circuit. Ph.D. Dissertation. Massachusetts Institute of Technology.

[7] Javier Hernandez, Yin Li, James M Rehg, and Rosalind W Picard. 2015. Cardiac and Respiratory Parameter Estimation Using Head-mounted Motion-sensitive Sensors. EAI Endorsed Trans. Pervasive Health Technol. 1, 1 (2015), e2.

[8] Javier Hernandez, Daniel McDuff, and Rosalind W Picard. 2015. Biowatch: estimation of heart and breathing rates from wrist motions. In 2015 9th International Conference on Pervasive Computing Technologies for Healthcare (PervasiveHealth). IEEE, 169-176.

[9] Fahim Kawsar, Chulhong Min, Akhil Mathur, and Allesandro Montanari. 2018. Earables for Personal-Scale Behavior Analytics. IEEE Pervasive Computing 17, 3 (2018), 83-89.

[10] JCY Leong, WW Lu, KDK Luk, and EM Karlberg. 1999. Kinematics of the chest cage and spine during breathing in healthy individuals and in patients with adolescent idiopathic scoliosis. Spine 24, 13 (1999), 1310.

[11] Alexis Martin and Jérémie Voix. 2017. In-ear audio wearable: Measurement of heart and breathing rates for health and safety monitoring. IEEE Transactions on Biomedical Engineering 65, 6 (2017), 1256-1263.

[12] Manuel Martinez and Rainer Stiefelhagen. 2012. Breath rate monitoring during sleep using near-IR imagery and PCA. In Proceedings of the 21st International Conference on Pattern Recognition (ICPR2012). IEEE, 3472-3475.

[13] Umesha Mogera, Abhay A Sagade, Subi J George, and Giridhar U Kulkarni. 2014. Ultrafast response humidity sensor using supramolecular nanofibre and its application in monitoring breath humidity and flow. Scientific reports 4 (2014), 4103.

[14] Yunyoung Nam, Bersain A Reyes, and Ki H Chon. 2015. Estimation of respiratory rates using the built-in microphone of a smartphone or headset. IEEE journal of biomedical and health informatics 20, 6 (2015), 1493-1501.

[15] Robert G Norman, Muhammed M Ahmed, Joyce A Walsleben, and David M Rapoport. 1997. Detection of respiratory events during NPSG: nasal cannula/pressure sensor versus thermistor. Sleep 20, 12 (1997), 1175-1184.

[16] Tobias Röddiger, Michael Beigl, Marcel Köpke, and Matthias Budde. 2018. VOCNEA: sleep apnea and hypopnea detection using a novel tiny gas sensor. In Proceedings of the 2018 ACM International Symposium on Wearable Computers. ACM, 226-227.

[17] Ameneh Shamekhi and Timothy Bickmore. 2018. Breathe Deep: A BreathSensitive Interactive Meditation Coach. In Proceedings of the 12th EAIInternational Conference on Pervasive Computing Technologies for Healthcare. ACM, 108-117.

[18] Xiao Sun, Li Qiu, Yibo Wu, Yeming Tang, and Guohong Cao. 2017. Sleepmonitor: Monitoring respiratory rate and body position during sleep using smartwatch. Proceedings of the ACM on Interactive, Mobile, Wearable and Ubiquitous Technologies 1, 3 (2017), 104

[19] Swaroop Venkatesh, Christopher R Anderson, Natalia V Rivera, and R Michael Buehrer. 2005. Implementation and analysis of respiration-rate estimation using impulse-based UWB. In MILCOM 2005-2005 IEEE Military Communications Conference. IEEE, 3314-3320.

[20] EJ Williams. 1949. Experimental designs balanced for the estimation of residual effects of treatments. Australian fournal of Chemistry 2, 2 (1949), 149-168.

[21] Jonathan D Witt, Jason RKO Fisher, Jordan A Guenette, Krystie A Cheong, Brock J Wilson, and A William Sheel. 2006. Measurement of exercise ventilation by a portable respiratory inductive plethysmograph. Respiratory physiology \& neurobiology 154, 3 (2006), 389-395. 
Karlsruher Institut für Technologie

\section{Repository KITopen}

Dies ist ein Postprint/begutachtetes Manuskript.

\section{Empfohlene Zitierung:}

Röddiger, T.; Wolffram, D.; Laubenstein, D.; Budde, M.; Beigl, M.

Towards Respiration Rate Monitoring Using an In-Ear Headphone Inertial Measurement Unit. 2019. UbiComp '19: The 2019 ACM International Joint Conference on Pervasive and Ubiquitous Computing - EarComp'19: Proceedings of the 1st International Workshop on Earable Computing - London, United Kingdom, 9th-13th September 2019, Association for Computing Machinery (ACM), New York (NY).

doi:10.5445/IR/1000118558

Zitierung der Originalveröffentlichung:

Röddiger, T.; Wolffram, D.; Laubenstein, D.; Budde, M.; Beigl, M.

Towards Respiration Rate Monitoring Using an In-Ear Headphone Inertial Measurement Unit. 2019. UbiComp '19: The 2019 ACM International Joint Conference on Pervasive and Ubiquitous Computing - EarComp'19: Proceedings of the 1st International Workshop on Earable Computing - London, United Kingdom, 9th-13th September 2019, 48-53, Association for Computing Machinery (ACM), New York (NY).

doi:10.1145/3345615.3361130 